\title{
Concurrent C: Safe Communities 09 A MODEL FOR EVALUATION OF SAFE COMMUNITIES IN LOW AND MIDDLE INCOME COUNTRIES
}

doi:10.1136/injuryprev-2012-040580e.9

\author{
${ }^{1,2,3} \mathrm{R}$ Mohammadi*, ${ }^{2} \mathrm{M}$ Zangi, ${ }^{1,3} \mathrm{H}$ Sadeghi-bazargani, ${ }^{2} \mathrm{~S}$ Hashemi. ${ }^{1}$ WHO \\ Collaborating Center on Community Safety Promotion, Karolinska Institutet, Sweden; \\ ${ }^{2}$ Municipality of Tehran, Iran; ${ }^{3}$ Injury Epidemiology \& Prevention Research Center, Tabriz \\ University of Medical Sciences, Iran
}

Background From all Safe Communities (SCs) indicators, evaluation is a neglected indicator especially in designated SCs in low and middle income (LMI) countries.

Aims/Objectives/Purpose Sets out to introduce a comprehensive model to carry out SC evaluation in LMI countries.

Methods Guidelines for safe community application as well as designated safe community reports were reviewed. Besides, Pubmed and Embase were searched to find scientific articles of injury prevention evaluation.

Results/Outcomes Three main approaches of SC evaluation can be summarised as follows: Firstly, process evaluation, in which, focus group discussion among reference groups of SC (work teams) is recommended. Secondly, impact evaluation, in which, quasi experimental study with emphasis on impact of interventions is most appropriate method to be conducted at designation and re-designation step between SC and control community. Knowledge, attitude, and practice (KAP) study, observational and survey are suggested for three main intervention; education, environmental change, enforcement of law respectively. The last but not the least, outcome evaluation, in which, quasi experimental study will be carried out.

Significance/Contribution to the Field Growing number of SC especially in LMI countries has given rise to the necessity of a comprehensive approach for SC evaluation in terms of process, impact and outcome. As an implication of this study a feasible evaluation model for Tehran safe community, as a model of safe communities in LMI countries, is designed and will be carried out after the implementation of the whole programme in the city. This evaluation plan and its results will be a strong tool for re-designation application after 5 years. 